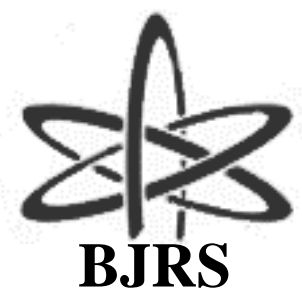

\author{
BRAZILIAN JOURNAL \\ $\mathrm{OF}$ \\ RADIATION SCIENCES \\ 08-03 (2020) 01-18
}

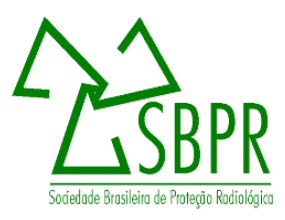

\title{
Simulation for non-homogeneous transport equation by Nyström method
}

\author{
Lazzari $^{\mathrm{a}}$ L., Sauter ${ }^{\mathrm{b}}$ E., De Azevedo ${ }^{\mathrm{c}}$ F. S. \\ ${ }^{a, b, c}$ Universidade Federal do Rio Grande do Sul, 91509900, Av. Bento Gonçalves, 9500 - Prédio 43111, Porto Alegre, \\ RS, Brazil \\ aluana-lazzari@hotmail.com \\ ${ }^{b}$ esequia.sauter@ufrgs.br \\ cfabio.azevedo@ufrgs.br
}

\begin{abstract}
In this work, we solve numerically the one-dimensional transport equation with semi-reflective boundary conditions and non-homogeneous domain. The proposed methodology consists of applying the Nyström method in order to discretize the integral formulation of this problem which is an equation involving weakly singular integral operators. For this purpose, analytical and computational techniques were applied to deal with the singularities. The Nyström method is an integral method that approximates the integral operator by a numerical quadrature and turns the integral equation into a finite dimensional linear system. This formulation allows us to use any function to describe both scattering cross section and total cross section. The algorithm is implemented in C language with the use of routines of GNU scientific library and computational techniques for code optimization. The scalar flux was calculated for two numerical quadrature, namely Gauss-Legendre quadrature and Boole's rule. The numerical results were determined for the transport problem with homogeneous and nonhomogeneous domains. In order to validate the proposed methodology, our numerical results were compared with those from the literature and presented with several correct significant digits.
\end{abstract}

Keywords: transport equation, integral formulation, Nyström method. 


\section{INTRODUCTION}

The transport equation has been the focus of many groups of researchers in recent years $[1,2$, 3]. One of the interests in this equation is to obtain a numerical solution for problems with different geometries and boundary conditions. Among the studied methodologies, we cite two large families: Discrete Ordinates [4] and Integral Methods [5]. The first family consist of discretizing the angular variable of the integro-differential transport equation producing a system of ordinary differential equations and, the second, consist of writing the equivalent integral equation where the angular variable does not appear explicitly, truncating the involved integral operators in matrices and producing a linear system.

Integral Methods, such as the Nyström method applied in this work, are known to produce accurate results for the transport equation in various geometries, since that the discretization is careful enough to remove the singularities from the kernel. Moreover, these methods work very well when the transport equation must be solved thousands of times with the same set of parameters and different sources. This happens in evolutionary problems, where the phenomenon of heat transfer involves diffusion, convection and radiation. In these cases, the transport equation is solved in the pre-processing and, at each step of time, for a new source, the scalar flux is obtained by a matrix multiplication [6]. Thus, although the Nyström method has a computational cost comparable to the methods in discrete ordinates to construct the matrices and solve the problem, there are those situations where it can really have a significant advantage in the simulation time without losing accuracy.

In this work, we apply the Nyström method to solve the one-dimensional transport equation in the steady-state, one-speed and non-homogeneous domain. Recently, Dalmolin et al. [7] simulated the transport problem with slab geometry and, Azevedo et al. [8] and Sauter et. al. [9] solved the same problem in X-Y geometry, both works applying the Nyström method, but none of them considered the non-homogeneous case. In fact, when the nuclear parameters depend on the spatial variable, some new complications appear as in obtaining the integral formulation as in discretizing the problem. 
The main idea in the Nyström method is to approach the integral operators in the equation applying a numerical quadrature and to solve a linear system for the scalar flux in the mesh points. However, the naive implementation of this methodology does not work because the kernel of the transport operator has a singularity. Here, we remove the singularity of the kernel and we make a careful implementation to produce a fast algorithm which calculates accurate results.

In order to validate the methodology, we compare our numerical results with those found in the literature. Results for the homogeneous, multilayer and non-homogeneous domains are compared with those presented by Dalmolin et al. [7], Nunes and Barros [10] and Garcia and Siewert [11] respectively. In the simulations we consider two numerical quadrature, namely Gauss-Legendre quadrature and Boole's rule. Also, tabulated convergence studies show good accuracy for scalar flux results with several correct significant digits.

The integro-differential formulation for the transport problem solved here is given by

$$
\mu \frac{\partial}{\partial x} \Psi(x, \mu)+\sigma_{t}(x) \Psi(x, \mu)=\frac{\sigma_{s}(x)}{2} \int_{-1}^{1} \Psi\left(x, \mu^{\prime}\right) d \mu^{\prime}+S(x)
$$

where $\Psi(x, \mu)$ is the angular flux, $0<x<L$ is the spatial variable and $\mu$ is the cosine of the angle formed between the direction of propagation and the axis $x$. The functions $\sigma_{t}(x)$ and $\sigma_{s}(x)$ represent the total macroscopic cross section and scattering macroscopic cross section which are different from zero and $\sigma_{t}(x)>\sigma_{s}(x)$. The function $S(x)$ is the isotropic internal source.

The semi-reflective boundary conditions considered here are given by

$$
\begin{aligned}
& \Psi(0, \mu)=\rho_{0}(\mu) \Psi(0,-\mu)+\left(1-\rho_{0}(\mu)\right) B_{0}(\mu), \mu>0, \\
& \Psi(L, \mu)=\rho_{L}(\mu) \Psi(L,-\mu)+\left(1-\rho_{L}(\mu)\right) B_{L}(\mu), \mu<0,
\end{aligned}
$$

where $0 \leq \rho_{0}, \rho_{L} \leq 1$ are the reflection coefficients and $B_{0}(\mu)$ and $B_{L}(\mu)$ represent the contribution of the boundary.

In view of the Nyström method is applied for the integral formulation of transport equation we first rewrite the problem as an integral equation and present calculus in Section 2. In Section 3 we 
present the discretization as well as the techniques to remove the singularities and implementation details. The numerical experiments are discussed in Section 4. We write the conclusion in Section 5.

\section{INTEGRAL FORMULATION}

In order to determine the integral form of the transport equation in non-homogeneous domain we rewrite the equation (1) as

$$
\frac{\partial}{\partial x} \Psi(x, \mu)+\frac{\sigma_{t}(x)}{\mu} \Psi(x, \mu)=\frac{Q(x)}{\mu},
$$

where $Q(x)=\sigma_{s}(x) \Phi(x)+S(x)$ and the scalar flux is defined by

$$
\Phi(x)=\frac{1}{2} \int_{-1}^{1} \Psi\left(x, \mu^{\prime}\right) d \mu^{\prime} .
$$

Then, we apply the integrating factor method in the differential equation (4) and we get

$$
\frac{\partial}{\partial x}\left(\Psi\left(x_{\nu} \mu\right) e^{\frac{1}{\mu} \int_{0}^{x} \sigma_{t}(k) d k}\right)=\frac{1}{\mu} e^{\frac{1}{\mu} \int_{0}^{x} \sigma_{t}(k) d k} Q(x) .
$$

We solve the equation (6) in two different ways, first integrating on $(0, \mathrm{x})$ and after on $(\mathrm{x}, \mathrm{L})$ and we obtain, respectively,

$$
\begin{gathered}
\Psi(x, \mu)=\Psi(0, \mu) e^{-\frac{1}{\mu} \int_{0}^{x} \sigma_{t}(k) d k}+\frac{1}{\mu} \int_{0}^{x} e^{-\frac{1}{\mu} \int_{\tau}^{x} \sigma_{t}(k) d k} Q(\tau) d \tau, \\
\Psi(x, \mu)=\Psi(L, \mu) e^{\frac{1}{\mu} \int_{x}^{L} \sigma_{t}(k) d k}-\frac{1}{\mu} \int_{x}^{L} e^{-\frac{1}{\mu} \int_{\tau}^{x} \sigma_{t}(k) d k} Q(\tau) d \tau .
\end{gathered}
$$

Here, both solutions are valid for $\mu>0$ and $\mu<0$ so we consider the expression (7) to the angular flux if $\mu>0$ and the equation (8) if $\mu<0$. We observe that the angular flux $\Psi(x, \mu)$ in 
equations (7) and (8) are not solved yet because $\Psi(0, \mu)$ and $\Psi(L, \mu)$ are not provided in all direction $\mu$ (see equations (2) and (3)).

In order to calculate the unknown fluxes at the boundary we consider $x=L$ in the equation (7), $x=0$ in the equation (8) and applying the boundary conditions (2) and (3) we have

$$
\begin{aligned}
& \Psi(L,-\mu)=\rho_{L}(-\mu) \Psi(0, \mu) e^{-\frac{1}{\mu} \int_{0}^{L} \sigma_{t}(k) d k}+\frac{\rho_{L}(-\mu)}{\mu} \int_{0}^{L} e^{-\frac{1}{\mu} \int_{\tau}^{L} \sigma_{t}(k) d k} Q(\tau) d \tau \\
& +\left(1-\rho_{\mathrm{L}}(-\mu)\right) \mathrm{B}_{\mathrm{L}}(-\mu), \quad \mu<0, \\
& \Psi(0, \mu)=\rho_{0}(\mu) \Psi(L,-\mu) e^{-\frac{1}{\mu} \int_{0}^{L} \sigma_{t}(k) d k}+\frac{\rho_{0}(\mu)}{\mu} \int_{0}^{L} e^{-\frac{1}{\mu} \int_{0}^{\tau} \sigma_{t}(k) d k} Q(\tau) d \tau \\
& +\left(1-\rho_{0}(\mu)\right) B_{0}(\mu), \quad \mu>0 .
\end{aligned}
$$

By equations (9) and (10) we obtain the linear system to the angular fluxes which are given by

$$
\begin{gathered}
\Psi(0, \mu)=\frac{\frac{\rho_{0}(\mu)}{\mu} \int_{0}^{L} e^{-\frac{1}{\mu} \int_{0}^{\tau} \sigma_{t}(k) d k} Q(\tau) d \tau+\left(1-\rho_{0}(-\mu)\right) B_{0}(\mu)}{1-\rho_{0}(\mu) \rho_{L}(-\mu) e^{-\frac{2}{\mu} \int_{0}^{L} \sigma_{t}(k) d k}} \\
+\frac{\frac{\rho_{0}(\mu) \rho_{L}(-\mu)}{\mu} e^{-\frac{1}{\mu} \int_{0}^{L} \sigma_{t}(k) d k} \int_{0}^{L} e^{-\frac{1}{\mu} \int_{\tau}^{L} \sigma_{t}(k) d k} Q(\tau) d \tau}{1-\rho_{0}(\mu) \rho_{L}(-\mu) e^{-\frac{2}{\mu} \int_{0}^{L} \sigma_{t}(k) d k}} \\
+\frac{\left(1-\rho_{L}(-\mu)\right) B_{L}(-\mu) \rho_{0}(\mu) e^{-\frac{1}{\mu} \int_{0}^{L} \sigma_{t}(k) d k}}{1-\rho_{0}(\mu) \rho_{L}(-\mu) e^{-\frac{2}{\mu} \int_{0}^{L} \sigma_{t}(k) d k}}
\end{gathered}
$$




$$
\begin{gathered}
\Psi(L, \mu)=\frac{\frac{\rho_{0}(\mu) \rho_{L}(-\mu)}{\mu} e^{-\frac{1}{\mu} \int_{0}^{L} \sigma_{t}(k) d k} \int_{0}^{L} e^{-\frac{1}{\mu} \int_{0}^{L} \sigma_{t}(k) d k} Q(\tau) d \tau}{1-\rho_{0}(\mu) \rho_{L}(-\mu) e^{-\frac{2}{\mu} \int_{0}^{L} \sigma_{t}(k) d k}} \\
+\frac{\frac{\rho_{L}(-\mu)}{\mu} \int_{0}^{L} e^{-\frac{1}{\mu} \int_{\tau}^{L} \sigma_{t}(k) d k} Q(\tau) d \tau+\left(1-\rho_{L}(-\mu)\right) B_{L}(-\mu)}{1-\rho_{0}(\mu) \rho_{L}(-\mu) e^{-\frac{2}{\mu} \int_{0}^{L} \sigma_{t}(k) d k}} \\
+\frac{\left(1-\rho_{0}(\mu)\right) B_{0}(\mu) \rho_{L}(-\mu) e^{-\frac{1}{\mu} \int_{0}^{L} \sigma_{t}(k) d k}}{1-\rho_{0}(\mu) \rho_{L}(-\mu) e^{-\frac{2}{\mu} \int_{0}^{L} \sigma_{t}(k) d k}} .
\end{gathered}
$$

Replacing the equation (11) in (7) we obtain

$$
\begin{aligned}
& \Psi(x, \mu)=\frac{e^{-\frac{1}{\mu} \int_{0}^{x} \sigma_{t}(k) d k}}{1-\rho_{0}(\mu) \rho_{L}(-\mu) e^{-\frac{2}{\mu} \int_{0}^{L} \sigma_{t}(k) d k}}\left[\frac{\rho_{0}(\mu)}{\mu} \int_{0}^{L} e^{-\frac{1}{\mu} \int_{0}^{T} \sigma_{t}(k) d k} Q(\tau) d \tau\right. \\
& +\frac{\rho_{0}(\mu) \rho_{L}(-\mu)}{\mu} e^{-\frac{1}{\mu} \int_{0}^{L} \sigma_{t}(k) d k} \int_{0}^{L} e^{-\frac{1}{\mu} \int_{\tau}^{L} \sigma_{t}(k) d k} Q(\tau) d \tau+\left(1-\rho_{0}(-\mu)\right) B_{0}(\mu) \\
& \left.+\left(1-\rho_{L}(-\mu)\right) B_{L}(-\mu) \rho_{0}(\mu) e^{-\frac{1}{\mu} \int_{0}^{L} \sigma_{t}(k) d k}\right]+\frac{1}{\mu} \int_{0}^{x} e^{-\frac{1}{\mu} \int_{\tau}^{x} \sigma_{t}(k) d k} Q(\tau) d \tau,
\end{aligned}
$$

and the equation (12) in (8), after changing $\mu$ for $-\mu$ in (8), we have

$$
\begin{aligned}
\Psi(x,-\mu)= & \frac{e^{-\frac{1}{\mu} \int_{x}^{L} \sigma_{t}(k) d k}}{1-\rho_{0}(\mu) \rho_{L}(-\mu) e^{-\frac{2}{\mu} \int_{0}^{L} \sigma_{t}(k) d k}}\left[\frac{\rho_{L}(-\mu)}{\mu} \int_{0}^{L} e^{-\frac{1}{\mu} \int_{\tau}^{L} \sigma_{t}(k) d k} Q(\tau) d \tau\right. \\
& +\frac{\rho_{0}(\mu) \rho_{L}(-\mu)}{\mu} e^{-\frac{1}{\mu} \int_{0}^{L} \sigma_{t}(k) d k} \int_{0}^{L} e^{-\frac{1}{\mu} \int_{0}^{\tau} \sigma_{t}(k) d k} Q(\tau) d \tau\left(1-\rho_{L}(-\mu)\right) B_{L}(-\mu) \\
& \left.+\left(1-\rho_{0}(\mu)\right) B_{0}(\mu) \rho_{L}(-\mu) e^{-\frac{1}{\mu} \int_{0}^{L} \sigma_{t}(k) d k}\right]+\frac{1}{\mu} \int_{x}^{L} e^{-\frac{1}{\mu} \int_{x}^{\tau} \sigma_{t}(k) d k} Q(\tau) d \tau .
\end{aligned}
$$

Now, we calculate the scalar flux $\Phi(x)$ integrating both expressions (13) and (14), the first on $[0,1)$ and, the second on $(-1,0]$. So we obtain, 


$$
\Phi(x)=\frac{1}{2} \int_{-1}^{1} \Psi(x, \mu) d \mu=\frac{1}{2} \int_{0}^{1}[\Psi(x,-\mu)+\Psi(x, \mu)] d \mu .
$$

The scalar flux in equation (15) combining with expressions (13) and (14) can be written in terms of two integral operator as follow,

$$
\Phi(x)=\left(L_{g} Q\right)(x)+\left(L_{b} B\right)(x),
$$

where the operator $\mathrm{L}_{\mathrm{g}}$ is given by

$$
L_{g} Q(x)=\int_{0}^{L} k(x, \tau) Q(\tau) d \tau,
$$

with kernel $k(x, \tau)$ expressed by

$$
\begin{gathered}
k(x, \tau)=\int_{0}^{1}\left[\frac{\rho_{L}(-\mu) e^{-\frac{1}{\mu}\left(\int_{x}^{L} \sigma_{t}(k) d k+\int_{\tau}^{L} \sigma_{t}(k) d k\right)}+\rho_{0}(\mu) e^{-\frac{1}{\mu}\left(\int_{0}^{T} \sigma_{t}(k) d k+\int_{0}^{x} \sigma_{t}(k) d k\right)}}{2 \mu\left(1-\rho_{0}(\mu) \rho_{L}(-\mu) e^{-\frac{2}{\mu} \int_{0}^{L} \sigma_{t}(k) d k}\right)}+\frac{e^{-\frac{1}{\mu}\left|\int_{x}^{T} \sigma_{t}(k) d k\right|}}{2 \mu}\right] \\
\\
\left.\quad+\frac{\rho_{0}(\mu) \rho_{L}(-\mu) e^{-\frac{2}{\mu} \int_{0}^{L} \sigma_{t}(k) d k}\left(e^{-\frac{1}{\mu} \int_{x}^{L} \sigma_{t}(k) d k}+e^{-\frac{1}{\mu} \int_{\tau}^{x} \sigma_{t}(k) d k}\right)}{2 \mu\left(1-\rho_{0}(\mu) \rho_{L}(-\mu) e^{-\frac{2}{\mu} \int_{0}^{L} \sigma_{t}(k) d k}\right)}\right] d \mu .
\end{gathered}
$$

The function $L_{b} B(x)$ is such that 


$$
\begin{aligned}
L_{b} B(x)=\int_{0}^{1}[ & \frac{\left.\left(1-\rho_{L}(-\mu)\right) B_{L}(-\mu) \rho_{0}(\mu) e^{-\frac{1}{\mu}\left(\int_{0}^{L} \sigma_{t}(k) d k+\int_{0}^{x} \sigma_{t}(k) d k\right.}\right)}{2\left(1-\rho_{0}(\mu) \rho_{L}(-\mu) e^{-\frac{2}{\mu} \int_{0}^{L} \sigma_{t}(k) d k}\right)} \\
& +\frac{\left(1-\rho_{0}(\mu)\right) B_{0}(\mu) e^{-\frac{1}{\mu} \int_{0}^{x} \sigma_{t}(k) d k}+\left(1-\rho_{L}(-\mu)\right) B_{L}(-\mu) e^{-\frac{1}{\mu} \int_{x}^{L} \sigma_{t}(k) d k}}{2\left(1-\rho_{0}(\mu) \rho_{L}(-\mu) e^{-\frac{2}{\mu} \int_{0}^{L} \sigma_{t}(k) d k}\right)} \\
& \left.+\frac{\left.\left(1-\rho_{0}(\mu)\right) B_{0}(\mu) \rho_{L}(-\mu) e^{-\frac{1}{\mu}\left(2 \int_{0}^{L} \sigma_{t}(k) d k-\int_{0}^{x} \sigma_{t}(k) d k\right.}\right)}{2\left(1-\rho_{0}(\mu) \rho_{L}(-\mu) e^{-\frac{2}{\mu} \int_{0}^{L} \sigma_{t}(k) d k}\right)}\right] d \mu .
\end{aligned}
$$

Replacing $Q(x)=\sigma_{s}(x) \Phi(x)+S(x)$ in the equation (16) we get the integral formulation to the onedimensional transport equation with non-homogeneous domain

$$
\Phi(x)=\int_{0}^{L} \sigma_{s}(\tau) k(x, \tau) \Phi(\tau) d \tau+g(x)
$$

where $g(x)=L_{g} S(x)+L_{b} B(x)$.

\section{DISCRETIZATION}

The function $k(x, \tau)$ in the equation (20) has a singularity when $\tau=x$. We apply the singularity subtraction technique in the kernel, i.e., $k(x, \tau) \Phi(\tau) \sigma_{s}(\tau)=k(x, \tau)\left[\Phi(\tau) \sigma_{s}(\tau)-\Phi(x) \sigma_{s}(x)\right]+k(x, \tau)$ $\Phi(x) \sigma_{s}(x)$ and we obtain the follow expression for the scalar flux:

$$
\Phi(x)=\int_{0}^{L} k(x, \tau)\left[\Phi(\tau) \sigma_{s}(\tau)-\Phi(x) \sigma_{s}(x)\right] d \tau+\Phi(x) \sigma_{s}(x) R(x)+g(x),
$$

where

$$
R(x)=\int_{0}^{L} k(x, \tau) d \tau
$$


We observe that the removal of singularity in equation (21) is essential for the stability of discretization. The Nyström method consists of approaching the integral term using a numerical quadrature, i.e.,

$$
\Phi(x) \approx \sum_{\substack{j=1 \\ \tau_{j} \neq x}}^{N} w\left(\tau_{j}\right) k\left(x, \tau_{j}\right)\left[\Phi\left(\tau_{j}\right) \sigma_{s}\left(\tau_{j}\right)-\Phi(x) \sigma_{s}(x)\right]+\Phi(x) \sigma_{s}(x) R(x)+g(x) .
$$

So, the scalar fluxes on the mesh points are approached by,

$$
\Phi_{i} \approx \sum_{\substack{j=1 \\ j \neq i}}^{N} w_{j} k_{i j}\left[\Phi_{j} \sigma_{s j}-\Phi_{i} \sigma_{s i}\right]+\Phi_{i} \sigma_{s i} R_{i}+g_{i}, \quad i=1, \ldots, N,
$$

where $\Phi\left(x_{i}\right)=\Phi_{i}, \sigma_{s}\left(x_{i}\right)=\sigma_{s i}, k\left(x_{i}, \tau_{j}\right)=k_{i j}, R\left(x_{i}\right)=R i, g\left(x_{i}\right)=g_{i}, w\left(\tau_{j}\right)=w_{j}$ are the quadrature weights and $x_{i}$ the nodes.

The code is implemented in $\mathrm{C}$ programming language and simulated on an Intel Core i7 computer. Observe that we have done an analytical treatment in the term $\mathrm{R}_{\mathrm{i}}$ to produce accurate results. Also, we solve integral terms using the numerical integration of the GNU Scientific Library (GSL). The linear system obtained from equation (24) is solved by LU decomposition method using a routine from GSL as well.

\section{NUMERICAL RESULTS}

In this section, we present the numerical results for the transport problem in homogeneous, multilayer and non-homogeneous domains with semi-reflective boundaries, given by equations (1)(3), using the methodology from Section 3. We present the results for two numerical quadrature schemes namely, Gauss-Legendre quadrature and Boole's rule. For each quadrature, we have done a convergence study in the mesh points, which allows us to compare the accuracy of the results, and 
for some cases, we present the computational time. We have chosen values of $\mathrm{N}$ between 1001 and 16001 and the numerical experiments have presented some correct significant digits.

In order to validate our results, we compare with the works of Nunes and Barros [10], Dalmolin et al. [7] and Garcia and Siewert [11]. Nunes and Barros presented the solution for the problems in homogeneous domain and multilayer slabs with three regions. These problems were solved with three numerical methods: Diamond Difference method (DD), Step method (S) and Step Characteristics method (SC). These methods were applied in the Discrete Ordinates Equation $\left(\mathrm{S}_{\mathrm{N}}\right)$ until order $S_{8}$. Dalmolin et al. [7] solved the homogeneous problem by Nyström method and obtained an accurate results for the scalar flux. The article by Garcia and Siewert [11] present simulations of radiative transfer problem in non-homogeneou domain whose solution is determined by the $\mathrm{F}_{\mathrm{N}}$ method.

\subsection{Homogeneous problem}

We start presenting results for the homogeneous problem introduced in [10] with the follow parameters: $L=50 \mathrm{~cm}, \rho_{0}(\mu)=\rho_{L}(\mu)=0, B_{0}(\mu)=B_{L}(\mu)=2, \sigma_{t}(x)=1 \mathrm{~cm}^{-1}, \sigma_{s}(x)=0.97 \mathrm{~cm}^{-1}$, and $S(x)=0$. Results are presented in Tables 1 and 2.

Table 1: Numerical results to Gauss-Legendre quadrature.

\begin{tabular}{ccccccccc}
\hline $\mathbf{x}$ & $\mathbf{D D}$ & $\mathbf{S}$ & $\mathbf{S C}$ & $\mathbf{N = 1 0 0 1}$ & $\mathbf{N = 2 0 0 1}$ & $\mathbf{N}=\mathbf{4 0 0 1}$ & $\mathbf{N = 8 0 0 1}$ & $\mathbf{N}=\mathbf{1 6 0 0 1}$ \\
\hline 0.0 & 1.704731 & 1.703453 & 1.704683 & 1.704732 & 1.704732 & 1.704732 & 1.704732 & 1.704732 \\
25.0 & 0.001956 & 0.002012 & 0.001958 & 0.001955 & 0.001955 & 0.001955 & 0.001955 & 0.001955 \\
50.0 & 1.704731 & 1.703435 & 1.704683 & 1.704732 & 1.704732 & 1.704732 & 1.704732 & 1.704732 \\
\hline
\end{tabular}

Table 2: Numerical results to Boole's rule.

\begin{tabular}{ccccccccc}
\hline $\mathbf{x}$ & $\mathbf{D D}$ & $\mathbf{S}$ & $\mathbf{S C}$ & $\mathbf{N}=\mathbf{1 0 0 1}$ & $\mathbf{N}=\mathbf{2 0 0 1}$ & $\mathbf{N}=\mathbf{4 0 0 1}$ & $\mathbf{N}=\mathbf{8 0 0 1}$ & $\mathbf{N}=\mathbf{1 6 0 0 1}$ \\
\hline 0.0 & 1.704731 & 1.703453 & 1.704683 & 1.704897 & 1.704778 & 1.704745 & 1.704735 & 1.704733 \\
25.0 & 0.001956 & 0.002012 & 0.001958 & 0.001955 & 0.001955 & 0.001955 & 0.001955 & 0.001955 \\
50.0 & 1.704731 & 1.703435 & 1.704683 & 1.704897 & 1.704778 & 1.704745 & 1.704735 & 1.704733 \\
\hline
\end{tabular}


In work [10] the Diamond Difference method presented the better accuracy when was compared DD, S and SD. Then we compared our solution with that from DD method [10]. The numerical results in the Tables 1 and 2 show a good numerical performance for both quadratures. We calculated the scalar flux with five correct significant digits using Gauss-Legendre quadrature and with the same precision applying Boole's rule. For the Gauss-Legendre quadrature the computational time to calculate the angular flux in $\mathrm{N}=1001, \mathrm{~N}=2001, \mathrm{~N}=4001, \mathrm{~N}=8001$ and $\mathrm{N}=16001$ was 23 s, $1 \mathrm{~m} 32$ s, $6 \mathrm{~m} 3$ s, $24 \mathrm{~m} 54$ s and 1 h $49 \mathrm{~m} 51$ s, respectively, and for the Boole's rule for the same values for $\mathrm{N}$ the computational time was $21 \mathrm{~s}, 1 \mathrm{~m} 25 \mathrm{~s}, 5 \mathrm{~m} 29 \mathrm{~s}, 23 \mathrm{~m} 53 \mathrm{~s}$ and $1 \mathrm{~h}$ $45 \mathrm{~m} 5 \mathrm{~s}$. We observed that, even with similar computational time, the Gauss-Legendre quadrature presents five correct digits with only 1001 points in the mesh while the Boole rule needs 8001 .

The second and third problems simulated here were proposed by Dalmolin et al. [7]. In that work the Nyström method was applied for solving the transport equation in homogeneous domain and semi-reflective boundaries. The numerical results presented in [7] were obtained using GaussLegendre quadrature and Boole's rule.

In the second problem we consider length domain $L=1 \mathrm{~cm}$, the reflection coefficients $\rho_{0}(\mu)=$ $\rho_{L}(\mu)=1$ and the boundary terms $B_{0}(\mu)=B_{L}(\mu)=1 / 8$. The cross sections are given by $\sigma_{t}(x)=$ $\sigma_{s}(x)=1 \mathrm{~cm}^{-1}$ and source term $S(x)=1 / 8$. In the third test, we consider length domain $L=1 \mathrm{~cm}$ and vacuum boundary conditions. The cross sections are given by $\sigma_{t}(x)=\sigma_{s}(x)=1 \mathrm{~cm}^{-1}$ and source term $S(x)=-x^{2}+x$. We simulated both problems using Gauss-Legendre quadrature and Boole's rule and the numerical results obtained here were the same presented by Dalmolin et al. [7]. These results were expected because the methodology applied by Dalmolin et al. [7] is the same used in this work, however it is restricted to homogeneous case.

\subsection{Multilayer slab problem}

The transport problem in multilayer slab was compared with the proposed by Nunes and Barros [10]. In that work, the numerical results were obtained by Diamond Difference method and were simulated for three regions. In this case we consider the problem with length domain $L=40 \mathrm{~cm}$ and boundary conditions $\Psi(0, \mu)=1$ and $\Psi(L, \mu)=1$. The functions $\sigma_{t}(x), \sigma_{s}(x)$, and $S(x)$ for each region are presented in Table 3. 
Table 3: Parameters of the non-homogeneous problem.

\begin{tabular}{cccc} 
Region & $\mathbf{1}$ & $\mathbf{2}$ & $\mathbf{3}$ \\
\hline$S(x)$ & 0 & 0 & 1 \\
$\sigma_{t}(x)$ & 1 & 1 & 1 \\
$\sigma_{s}(x)$ & 0.97 & 0.95 & 0.99 \\
Thickness & 15 & 15 & 10 \\
\hline
\end{tabular}

Table 4: Numerical results to Gauss-Legendre quadrature.

\begin{tabular}{cccccccc}
\hline $\mathbf{x}$ & $\mathbf{D D}$ & $\mathbf{N}=\mathbf{5 0 1}$ & $\mathbf{N}=\mathbf{1 0 0 1}$ & $\mathbf{N}=\mathbf{2 0 0 1}$ & $\mathbf{N}=\mathbf{4 0 0 1}$ & $\mathbf{N}=\mathbf{8 0 0 1}$ & $\mathbf{N}=\mathbf{1 6 0 0 1}$ \\
\hline 0.0 & 0.852638 & 0.852638 & 0.852638 & 0.852638 & 0.852638 & 0.852638 & 0.852638 \\
20.0 & 0.481766 & 0.482850 & 0.484256 & 0.482214 & 0.482542 & 0.482026 & 0.482108 \\
40.0 & 7.090416 & 7.093101 & 7.095029 & 7.091948 & 7.092451 & 7.091686 & 7.091813 \\
\hline
\end{tabular}

Table 5: Numerical results to Boole's rule.

\begin{tabular}{cccccccc}
\hline $\mathbf{x}$ & $\mathbf{D D}$ & $\mathbf{N = 5 0 1}$ & $\mathbf{N}=\mathbf{1 0 0 1}$ & $\mathbf{N}=\mathbf{2 0 0 1}$ & $\mathbf{N}=\mathbf{4 0 0 1}$ & $\mathbf{N}=\mathbf{8 0 0 1}$ & $\mathbf{N}=\mathbf{1 6 0 0 1}$ \\
\hline 0.0 & 0.852638 & 0.852831 & 0.852693 & 0.852653 & 0.852642 & 0.852639 & 0.852638 \\
20.0 & 0.481766 & 0.476498 & 0.480935 & 0.481354 & 0.481662 & 0.481815 & 0.481892 \\
40.0 & 7.090416 & 7.073885 & 7.087380 & 7.089942 & 7.090936 & 7.091313 & 7.091469 \\
\hline
\end{tabular}

In the Tables 4 and 5 are tabulated the numerical results for the transport problem in multilayer slab. We observe that the solution presented in work [9] were simulated with angular discretization of order $\mathrm{S}_{8}$ which is low to obtain good accuracy. The numerical results of this work presented one or two digits while our methodology produced results with six significant digits. We approach the integral operators using Gauss-Legendre quadrature and Boole's rule and both quadratures had similar performance when the number of nodes is large. The computational time to determine the solution of the problem in multilayer slab at $\mathrm{N}=501, \mathrm{~N}=1001, \mathrm{~N}=2001, \mathrm{~N}=4001, \mathrm{~N}=8001$ and $\mathrm{N}=1601$ was $7 \mathrm{~s}, 25 \mathrm{~s}, 1 \mathrm{~m} 36 \mathrm{~s}, 6 \mathrm{~m} 22 \mathrm{~s}, 25 \mathrm{~m} 9 \mathrm{~s}$ and $1 \mathrm{~h} 55 \mathrm{~m} 34 \mathrm{~s}$ for the Gauss-Legendre quadrature and $7 \mathrm{~s}, 24 \mathrm{~s}, 1 \mathrm{~m} 30 \mathrm{~s}, 6 \mathrm{~m} 3 \mathrm{~s}, 25 \mathrm{~m} 54 \mathrm{~s}$ and $1 \mathrm{~h} 51 \mathrm{~m} 58 \mathrm{~s}$ when we apply Boole's rule. 
therefore, when we consider the same number of points in the mesh, the computational performance of the quadradures is similar.

\subsection{Non-homogenenous problem}

Finally, we validate our results comparing them with that obtained by Garcia and Siewert [11]. In this work the authors determined the exit distributions, $\Psi(0,-\mu)$ and $\Psi(L, \mu)$, of the radiative transfer problem in non-homogeneous domain with an exponentially varying albedo by $F_{N}$ method. In order to compare our numerical results with the solution by Garcia and Siewert [11] we determinate the exit distribution from the scalar flux as in the following equations,

$$
\begin{aligned}
\Psi(0,-\mu)= & \int_{0}^{L}\left\{\frac { 1 } { 1 - \rho _ { 0 } ( \mu ) \rho _ { L } ( - \mu ) e ^ { - \frac { 2 L } { \mu } } } \left[\frac{\rho_{0}(\mu) \rho_{L}(-\mu) e^{-\frac{1}{\mu}(2 L-\tau)}}{\mu}\left[\sigma_{s}(\tau) \Phi(\tau)+S(\tau)\right]\right.\right. \\
& \left.\left.+\frac{\rho_{L}(-\mu) e^{-\frac{1}{\mu}(2 L+\tau)}}{\mu}\left[\sigma_{s}(\tau) \Phi(\tau)+S(\tau)\right]\right]+\frac{e^{-\frac{\tau}{\mu}}}{\mu}\left[\sigma_{s}(\tau) \Phi(\tau)+S(\tau)\right]\right\} d \tau \\
& +\frac{1-\rho_{L}(\mu) B_{L}(\mu) e^{-\frac{L}{\mu}}+\left(1-\rho_{0}(\mu)\right) B_{0}(\mu) \rho_{L}(\mu) e^{-\frac{2 L}{\mu}}}{1-\rho_{0}(\mu) \rho_{L}(-\mu) e^{-\frac{2 L}{\mu}}}
\end{aligned}
$$

and

$$
\begin{aligned}
& \Psi(L, \mu)=\int_{0}^{L}\left\{\frac { 1 } { 1 - \rho _ { 0 } ( \mu ) \rho _ { L } ( - \mu ) e ^ { - \frac { 2 L } { \mu } } } \left[\frac{\rho_{0}(\mu) \rho_{L}(\mu) e^{-\frac{1}{\mu}(3 L-\tau)}}{\mu}\left[\sigma_{s}(\tau) \Phi(\tau)+S(\tau)\right]\right.\right. \\
&\left.+\frac{\rho_{0}(\mu) e^{-\frac{1}{\mu}(L+\tau)}}{\mu}\left[\sigma_{s}(\tau) \Phi(\tau)+S(\tau)\right]+\frac{e^{-\frac{1}{\mu}(L-\tau)}}{\mu}\left[\sigma_{s}(\tau) \Phi(\tau)+S(\tau)\right]\right\} d \tau \\
&+\frac{1-\rho_{0}(\mu) B_{0}(\mu) e^{-\frac{L}{\mu}}+\left(1-\rho_{L}(-\mu)\right) B_{L}(-\mu) \rho_{0}(\mu) e^{-\frac{2 L}{\mu}}}{1-\rho_{0}(\mu) \rho_{L}(-\mu) e^{-\frac{2 L}{\mu}}}
\end{aligned}
$$


In the first problem simulated we consider $L=5 \mathrm{~cm}, \rho_{0}(\mu)=\rho_{L}(\mu)=B_{L}(\mu)=0, B_{0}(\mu)=1$, $\sigma_{t}(x)=1 \mathrm{~cm}^{-1}, \sigma_{s}(x)=e^{-x / s} \mathrm{~cm}^{-1}$, and $S(x)=0$. The numerical results for the exit distribution are presenteds in the Tables 5 and 6 .

Table 5: The exit distribution $\Psi(0,-\mu)$.

\begin{tabular}{ccccccc}
\hline $\boldsymbol{\mu}$ & $\mathbf{S}^{1} \mathbf{1}^{[\mathbf{1 0}]}$ & $\mathbf{S}=\mathbf{1}$ & $\mathbf{S}=\mathbf{1 0}^{[\mathbf{1 0}]}$ & $\mathbf{S}=\mathbf{1 0}$ & $\mathbf{S}=\mathbf{1 0 0}^{[\mathbf{1 0}]}$ & $\mathbf{S}=\mathbf{1 0 0}$ \\
\hline 0.05 & 0.58966 & 0.58966 & 0.76081 & 0.76081 & 0.86400 & 0.86400 \\
0.1 & 0.53112 & 0.53112 & 0.73398 & 0.73398 & 0.85028 & 0.85028 \\
0.2 & 0.44328 & 0.44328 & 0.68632 & 0.68632 & 0.82523 & 0.82523 \\
0.3 & 0.38031 & 0.38031 & 0.64418 & 0.64418 & 0.80181 & 0.80181 \\
0.4 & 0.33296 & 0.33296 & 0.60647 & 0.60647 & 0.77946 & 0.77946 \\
0.5 & 0.29609 & 0.29609 & 0.57257 & 0.57257 & 0.75799 & 0.75799 \\
0.6 & 0.26656 & 0.26656 & 0.54197 & 0.54197 & 0.73730 & 0.73730 \\
0.7 & 0.24239 & 0.24239 & 0.51427 & 0.51427 & 0.71733 & 0.71733 \\
0.8 & 0.22223 & 0.22223 & 0.48910 & 0.48910 & 0.69805 & 0.69805 \\
0.9 & 0.20517 & 0.20517 & 0.46615 & 0.46615 & 0.67943 & 0.67943 \\
1.0 & 0.19055 & 0.19055 & 0.44517 & 0.44517 & 0.66146 & 0.66146 \\
\hline
\end{tabular}

Table 6: The exit distribution $\Psi(L, \mu)$.

\begin{tabular}{ccccccc}
\hline $\boldsymbol{\mu}$ & ${\mathbf{s}=\mathbf{1}^{[10]}}$ & $\mathbf{s}=\mathbf{1}$ & $\mathbf{S}=\mathbf{1 0}^{[10]}$ & $\mathbf{s}=\mathbf{1 0}$ & $\mathbf{s}=\mathbf{1 0 0}^{[10]}$ & $\mathbf{s}=\mathbf{1 0 0}$ \\
\hline 0.05 & $0.6075 \mathrm{E}-5$ & $0.6075 \mathrm{E}-5$ & $0.58031 \mathrm{E}-2$ & $0.58031 \mathrm{E}-2$ & $0.62883 \mathrm{E}-1$ & $0.62883 \mathrm{E}-1$ \\
0.1 & $0.69252 \mathrm{E}-5$ & $0.69252 \mathrm{E}-5$ & $0.63702 \mathrm{E}-2$ & $0.63702 \mathrm{E}-2$ & $0.69024 \mathrm{E}-1$ & $0.69024 \mathrm{E}-1$ \\
0.2 & $0.96423 \mathrm{E}-5$ & $0.96423 \mathrm{E}-5$ & $0.76183 \mathrm{E}-2$ & $0.76183 \mathrm{E}-2$ & $0.80567 \mathrm{E}-1$ & $0.80567 \mathrm{E}-1$ \\
0.3 & $0.16234 \mathrm{E}-4$ & $0.16234 \mathrm{E}-4$ & $0.91482 \mathrm{E}-2$ & $0.91482 \mathrm{E}-2$ & $0.91918 \mathrm{E}-1$ & $0.91918 \mathrm{E}-1$ \\
0.4 & $0.43858 \mathrm{E}-4$ & $0.43858 \mathrm{E}-4$ & $0.11119 \mathrm{E}-1$ & $0.11119 \mathrm{E}-1$ & 0.14012 & 0.14012 \\
0.5 & $0.16937 \mathrm{E}-3$ & $0.16937 \mathrm{E}-3$ & $0.13725 \mathrm{E}-1$ & $0.13725 \mathrm{E}-1$ & 0.11523 & 0.11523 \\
0.6 & $0.57347 \mathrm{E}-3$ & $0.57347 \mathrm{E}-3$ & $0.17183 \mathrm{E}-1$ & $0.17183 \mathrm{E}-1$ & 0.12744 & 0.12744 \\
0.7 & $0.15128 \mathrm{E}-2$ & $0.15128 \mathrm{E}-2$ & $0.21680 \mathrm{E}-1$ & $0.21680 \mathrm{E}-1$ & 0.14010 & 0.14010 \\
0.8 & $0.32437 \mathrm{E}-2$ & $0.32437 \mathrm{E}-2$ & $0.27331 \mathrm{E}-1$ & $0.27331 \mathrm{E}-1$ & 0.15319 & 0.15319 \\
0.9 & $0.59604 \mathrm{E}-2$ & $0.59604 \mathrm{E}-2$ & $0.34166 \mathrm{E}-1$ & $0.34166 \mathrm{E}-1$ & 0.16667 & 0.16667 \\
\hline
\end{tabular}


$\begin{array}{lllllll}1.0 & 0.97712 \mathrm{E}-2 & 0.97712 \mathrm{E}-2 & 0.42142 \mathrm{E}-1 & 0.42142 \mathrm{E}-1 & 0.18047 & 0.18047\end{array}$

In the second problem we consider the follow parameters : $L=5 m, \rho_{0}(\mu)=\rho_{L}(\mu)=B_{L}(\mu)=0$, $B_{0}(\mu)=\delta(\mu-0.9), \sigma_{t}(x)=1 \mathrm{~cm}^{-1}, \sigma_{s}(x)=e^{-x / s} \mathrm{~cm}^{-1}$ and $S(x)=0$. Results are presented in Tables 7 and 8.

Table 7: The exit distribution $\Psi(0,-\mu)$.

\begin{tabular}{ccccccc}
\hline $\boldsymbol{\mu}$ & $\mathbf{S = 1}^{[\mathbf{1 0}]}$ & $\mathbf{S}=\mathbf{1}$ & $\mathbf{S}=\mathbf{1 0}^{[\mathbf{1 0}]}$ & $\mathbf{S}=\mathbf{1 0}$ & $\mathbf{s}=\mathbf{1 0 0}^{[\mathbf{1 0}]}$ & $\mathbf{S}=\mathbf{1 0 0}$ \\
\hline 0.05 & 0.69801 & 0.69801 & 0.98398 & 0.98398 & $0.11842 \mathrm{E}-1$ & $0.11842 \mathrm{E}-1$ \\
0.1 & 0.65651 & 0.65651 & 0.99343 & 0.99343 & $0.12182 \mathrm{E}-1$ & $0.12182 \mathrm{E}-1$ \\
0.2 & 0.57637 & 0.57637 & 0.98472 & 0.98472 & $0.12531 \mathrm{E}-1$ & $0.12531 \mathrm{E}-1$ \\
0.3 & 0.50955 & 0.50955 & 0.95989 & 0.95989 & $0.12655 \mathrm{E}-1$ & $0.12655 \mathrm{E}-1$ \\
0.4 & 0.45523 & 0.45523 & 0.92849 & 0.92849 & $0.12655 \mathrm{E}-1$ & $0.12655 \mathrm{E}-1$ \\
0.5 & 0.41078 & 0.41078 & 0.89472 & 0.89472 & $0.12655 \mathrm{E}-1$ & $0.12655 \mathrm{E}-1$ \\
0.6 & 0.37396 & 0.37396 & 0.86064 & 0.86064 & $0.12655 \mathrm{E}-1$ & $0.12655 \mathrm{E}-1$ \\
0.7 & 0.34304 & 0.34304 & 0.86064 & 0.86064 & $0.12655 \mathrm{E}-1$ & $0.12655 \mathrm{E}-1$ \\
0.8 & 0.31676 & 0.31676 & 0.79531 & 0.79531 & $0.12089 \mathrm{E}-1$ & $0.12089 \mathrm{E}-1$ \\
0.9 & 0.29416 & 0.29416 & 0.76486 & 0.76486 & $0.11883 \mathrm{E}-1$ & $0.11883 \mathrm{E}-1$ \\
1.0 & 0.27454 & 0.27454 & 0.73605 & 0.73605 & $0.11665 \mathrm{E}-1$ & $0.11665 \mathrm{E}-1$ \\
\hline
\end{tabular}

Table 8: The exit distribution $\Psi(L, \mu)$.

\begin{tabular}{ccccccc}
\hline $\boldsymbol{\mu}$ & $\mathbf{S}^{2} \mathbf{1}^{[\mathbf{1 0}]}$ & $\mathbf{S}=\mathbf{1}$ & $\mathbf{s = 1 0}$ & $\mathbf{S}=\mathbf{1 0}$ & $\mathbf{S}=\mathbf{1 0 0}^{[\mathbf{1 0 0}]}$ & $\mathbf{s = 1 0 0}$ \\
\hline 0.05 & $0.18678 \mathrm{E}-4$ & $0.18678 \mathrm{E}-4$ & $0.13216 \mathrm{E}-1$ & $0.13216 \mathrm{E}-1$ & 0.13460 & 0.13460 \\
0.1 & $0.21226 \mathrm{E}-4$ & $0.21226 \mathrm{E}-4$ & $0.14498 \mathrm{E}-1$ & $0.14498 \mathrm{E}-1$ & 0.14772 & 0.14772 \\
0.2 & $0.29214 \mathrm{E}-4$ & $0.29214 \mathrm{E}-4$ & $0.17307 \mathrm{E}-1$ & $0.17307 \mathrm{E}-1$ & 0.17237 & 0.17237 \\
0.3 & $0.47082 \mathrm{E}-4$ & $0.47082 \mathrm{E}-4$ & $0.20723 \mathrm{E}-1$ & $0.20723 \mathrm{E}-1$ & 0.19656 & 0.19656 \\
0.4 & $0.10218 \mathrm{E}-3$ & $0.10218 \mathrm{E}-3$ & $0.25054 \mathrm{E}-1$ & $0.25054 \mathrm{E}-1$ & 0.22097 & 0.22097 \\
0.5 & $0.26855 \mathrm{E}-3$ & $0.26855 \mathrm{E}-3$ & $0.30578 \mathrm{E}-1$ & $0.30578 \mathrm{E}-1$ & 0.24579 & 0.24579 \\
0.6 & $0.65035 \mathrm{E}-3$ & $0.65035 \mathrm{E}-3$ & $0.37422 \mathrm{E}-1$ & $0.37422 \mathrm{E}-1$ & 0.27089 & 0.27089 \\
0.7 & $0.13276 \mathrm{E}-2$ & $0.13276 \mathrm{E}-2$ & $0.45484 \mathrm{E}-1$ & $0.45484 \mathrm{E}-1$ & 0.29586 & 0.29586 \\
0.8 & $0.23249 \mathrm{E}-2$ & $0.23249 \mathrm{E}-2$ & $0.54487 \mathrm{E}-1$ & $0.54487 \mathrm{E}-1$ & 0.32019 & 0.32019 \\
\hline
\end{tabular}




$\begin{array}{ccccccc}0.9 & 0.36161 \mathrm{E}-2 & 0.36161 \mathrm{E}-2 & 0.64073 \mathrm{E}-1 & 0.64073 \mathrm{E}-1 & 0.34339 & 0.34339 \\ 1.0 & 0.51444 \mathrm{E}-2 & 0.51444 \mathrm{E}-2 & 0.73894 \mathrm{E}-1 & 0.73894 \mathrm{E}-1 & 0.36507 & 0.36507\end{array}$

In the Tables 5, 6, 7 and 8 we tabulate the results numerical for the exit distributions of the radiative transfer problem in non-homogeneous domain with an exponentially varying albedo. Our solution presented five digits of precision as well as in the paper of Garcia and Siewert [11]. In all simulations, the solution converged more quickly when we approach the integral operator using the Gauss-Legendre quadrature, in some cases it was up to sixteen times faster than the Boole's rule.

For the case where $\mathrm{s}=100$ in Table 5, we obtained five digits of precision at $\mathrm{N}=401$ points when applying the Gauss-Legendre quadrature and the same precision when considering $\mathrm{N}=801$ points for Boole's rule. For the Gauss-Legendre quadrature, the computational time to determine the exit distribution for $\mathrm{N}=101, \mathrm{~N}=201, \mathrm{~N}=401$ and $\mathrm{N}=801$ was $27 \mathrm{~s}, 1 \mathrm{~m} 37 \mathrm{~s}, 6 \mathrm{~m} 23 \mathrm{~s}$ and $25 \mathrm{~m}$ $41 \mathrm{~s}$, respectively, when we apply the Boole's rule we obtain the following computational times 27 s, $1 \mathrm{~m} 41 \mathrm{~s}, 6 \mathrm{~m} 29 \mathrm{~s}$ and $25 \mathrm{~m} 44 \mathrm{~s}$ for the same number of points. Although the solution converging more quickly when we apply the Gauss-Legendre quadrature we have that the computational time is similar when we simulate both quadratures for the same number of points in the mesh. This same behavior is repeated in all other simulations.

\section{CONCLUSION}

In this work, we determined the numerical solution for the one-dimensional transport equation with non-homogeneous domain and semi-reflective boundaries by Nyström method. For being an integral method we worked with formulation integral of this problem associated with the application of techniques to remove the singularities of the kernel of the integral operator. The removal of singularity was essential for the stability of discretization of the problem by Nyström method.

In order to check the efficiency of the methodology proposed in this work we resolved problems in homogeneous, multilayer and non-homogeneous domains using Gauss-Legendre quadrature and Boole's rule. The numerical results produced in the simulations presented the same precision that 
literature data and in some cases were more accurate like in the multilayer problem. Analyzing the performance of the quadratures, we observe that both presented similar behavior when the number of nodes is large.

Our code is numerically efficient because it produced accurate results with low computational cost. It is also robust because it solved problems in homogeneous and non-homogeneous domains, including multilayer and more general cases.

\section{ACKNOWLEDGMENT}

L. Lazzari was supported by a doctoral fellowship of the CAPES (Brazil).

\section{REFERENCES}

[1] BARICHELLO, L. B.; SIEWERT C. E. A new version of the discrete-ordinates method. In: Proc. of the 2nd Conf. on Comput. Heat and Mass Transfer (COPPE/EE/UFRJ), Rio de Janeiro, Brazil, 2001.

[2] DOMÍNGUEZ, D. S.; BARROS, R. C. The spectral Green's function linear-nodal method for one-speed X, Y-geometry discrete ordinates deep penetration problems. Annals of Nuclear Energy, vol. 34, p. 958-966, 2007.

[3] SAUTER, E.; DE AZEVEDO, F.; THOMPSON, M.; VILHENA, M.T. Solution of the onedimensional transport equation by the vector green function method: Error bounds and simulation. Applied Mathematics and Computation, p. 11291-11301, 2013.

[4] CHANDRASEKHAR, S. Radiative transfer, Dover Publications, Inc., Oxford University Press, London, 1950.

[5] LEWIS, E. E.; MILLER, J. W. F. Computational Methods of Neutron Transport, Copyright, United States of America, 1984. 
[6] DE AZEVEDO, F. S.; SAUTER, E.; THOMPSON, M. Existence theory and simulations for one-dimensional radiative flows. Annals of Nuclear Energy. vol. 38, p. 1115-1124, 2010.

[7] DALMOLIN, D.; DE AZEVEDO F. S.; SAUTER, E. Nyström method in transport equation, PROCEEDING OF INAC 2017 INTERNATIONAL NUCLEAR ATLANTIC CONFERENCE, Rio de Janeiro, Brazil, 2017.

[8] DE AZEVEDO, F. S.; SAUTER, E.; KONZEN, P. H. A.; THOMPSON, M.; BARICHELLO, L. B. Integral formulation and numerical simulations for the neutron transport equation in $\mathrm{X}-\mathrm{Y}$ geometry. Annals of Nuclear Energy, vol. 112, p. 735-747, 2018.

[9] SAUTER, E.; DE AZEVEDO, F. S.; KONZEN, P. H. A. Nyström Method Applied to the Transport Equation in a Semi-Reflective Rectangle. Journal of Computational and Theoretical Transport, p. 2332-4325, 2019.

[10] NUNES, C. E. A.; BARROS, R. C. Aplicativo computacional para cálculos de blindagem com modelo de transporte $S_{n}$ unidimensional e monoenergético, PROCEEDING OF INAC 2009 INTERNATIONAL NUCLEAR ATLANTIC CONFERENCE, Rio de Janeiro, Brazil, 2009.

[11] GARCIA, R. D. M.; SIEWERT, C. E. Radiative transfer in finite inhomogeneous planeparallel atmospheres. Journal of Quantitative Spectroscopy e Radiative Transfer, 2009. 